The Astrophysical Journal, 648:1276-1284, 2006 September 10

(C) 2006. The American Astronomical Society. All rights reserved. Printed in U.S.A.

\title{
CALIBRATING AN INTERFEROMETRIC NULL
}

\author{
BENJAMIN F. LaNe \\ Kavli Institute for Astrophysics and Space Research, Department of Physics, Massachusetts Institute of Technolgy 70 Vassar Street, \\ Cambridge, MA 02139; blane@mit.edu \\ Matthew W. Muterspaugh \\ Department of Geological and Planetary Sciences, California Institute of Technology, Pasadena, CA 91125; matthew1@mit.edu
}

AND

Michael Shao

Jet Propulsion Laboratory, California Institute of Technology, 4800 Oak Grove Drive, Pasadena, CA 91109; mshao@huey.jpl.nasa.gov Received 2006 April 10; accepted 2006 May 20

\begin{abstract}
One of the biggest challenges associated with a nulling-interferometer-based approach to detecting extrasolar Earthlike planets comes from the extremely stringent requirements of path length, polarization, and amplitude matching in the interferometer. To the extent that the light from multiple apertures is not matched in these properties, light will leak through the nuller and confuse the search for a planetary signal. Here we explore the possibility of using the coherence properties of the starlight to separate contributions from the planet and nuller leakage. We find that straightforward modifications to the optical layout of a nulling interferometer will allow one to measure and correct for the leakage to a high degree of precision. This nulling calibration relaxes the field matching requirements substantially and should consequently simplify the instrument design.
\end{abstract}

Subject heading: techniques: interferometric

\section{INTRODUCTION}

One suggested method for finding Earth-like planets orbiting other stars involves a space-based nulling interferometer operating in the thermal IR (Bracewell 1978; Woolf \& Angel 1998; Noecker 1999). NASA has been considering such a mission: Terrestrial Planet Finder (TPF; Coulter 2004), while ESA is planning an equivalent mission: Darwin (Kaltenegger et al. 2003). The primary challenge in detecting such planets is the extreme contrast ratio between the planet and its primary star $\left(\sim 10^{6}-10^{7}\right.$ at a wavelength of $10 \mu \mathrm{m})$ and close angular separation $\left(\sim 00^{\prime \prime} 1\right)$. To prevent the planet light from being overwhelmed by photon noise from the primary, some way of selectively nulling the starlight is required. However, the extreme nulling ratio desired sets very stringent limits on the allowable instrument performance, specifically in terms of path length error $\left(\delta \phi^{2}\right)$, polarization $\left(\delta \alpha^{2}\right)$, and amplitude mismatch $\left(\delta a^{2}\right)$, all of which cause "leakage" in the null. Lay (2004) has shown that for certain nulling configurations, in addition to simple leakage terms of the form $\delta \phi^{2}$ or $\delta a^{2}$, second-order coupling effects of the form $\delta \phi \delta a$ lead to stability requirements on the order of $\sim 1.5 \mathrm{~nm}$ in path and $\sim 0.1 \%$ in amplitude over hour-long timescales. These performance levels are almost an order of magnitude more stringent than previous estimates, and pose severe challenges to the $T P F$ mission design.

In this paper we explore a simple way of determining the nulling leakage based on exploiting the fact that the leakage light is coherent with the starlight, but not with the planet light. By making a simple modification to the interferometer back end it becomes possible to measure the leakage terms and hence calibrate the null. This idea is an adaptation of the "synchronous interferometric speckle subtraction" concept proposed by Guyon (2004) for coronagraphic instruments. In $\S 2$ we describe the original Bracewell nuller concept. We then introduce the nulling calibration technique and derive its expected performance, as well as show simple simulations of the application. In $\S 3$ we introduce the more complicated dual Bracewell architecture actually being considered for $T P F$, as well as show how the calibration concept could be applied to it. We discuss the effects this might have on the TPF system design in $\S 4$.

\section{THE SINGLE BRACEWELL NULLER}

Consider Figure 1. Light is collected from two apertures separated by a baseline $\boldsymbol{B}$ and brought to a common point such that the wave fronts from the two arms are exactly $\pi / 2$ out of phase with respect to each other going into the beam splitter, for a total postcombination phase shift of $\pi$. Recall that a beam splitter introduces an additional $\pi / 2$ relative phase shift between reflected and transmitted beams. For a monochromatic interferometer the initial $\pi / 2$ is simply a path adjustment. However, in the more realistic case of a broadband interferometer a more sophisticated approach becomes necessary, typically in the form of "phase plates;" i.e., one beam is passed through a set of carefully controlled thicknesses of glass arranged such that the wavelength-dependent indices of refraction combine to give a $\pi / 2$ phase shift across a $10 \%-20 \%$ bandpass (Wallace et al. 2004). At the 50/50 beam splitter the two beams are combined, and the intensities can be measured at the two complementary outputs. Note that the planet-star separation is unresolved by the individual apertures, and hence in this "pupil plane" interferometer, detection of the planet signal requires that it be modulated in some fashion; in the Bracewell configuration this is accomplished by rotating the interferometer about the axis pointing to the star.

If we neglect time dependence and polarization of the electric fields, we can write

$$
\begin{gathered}
E_{1}=A a_{1} e^{i\left(\phi_{1}\right)}, \\
E_{2}=A a_{2} e^{i\left(\phi_{2}+\pi / 2\right)},
\end{gathered}
$$

where $A$ is the amplitude of the field seen by an ideal aperture and $\phi_{1}, \phi_{2}, a_{1}$, and $a_{2}$ represent small phase and amplitude 


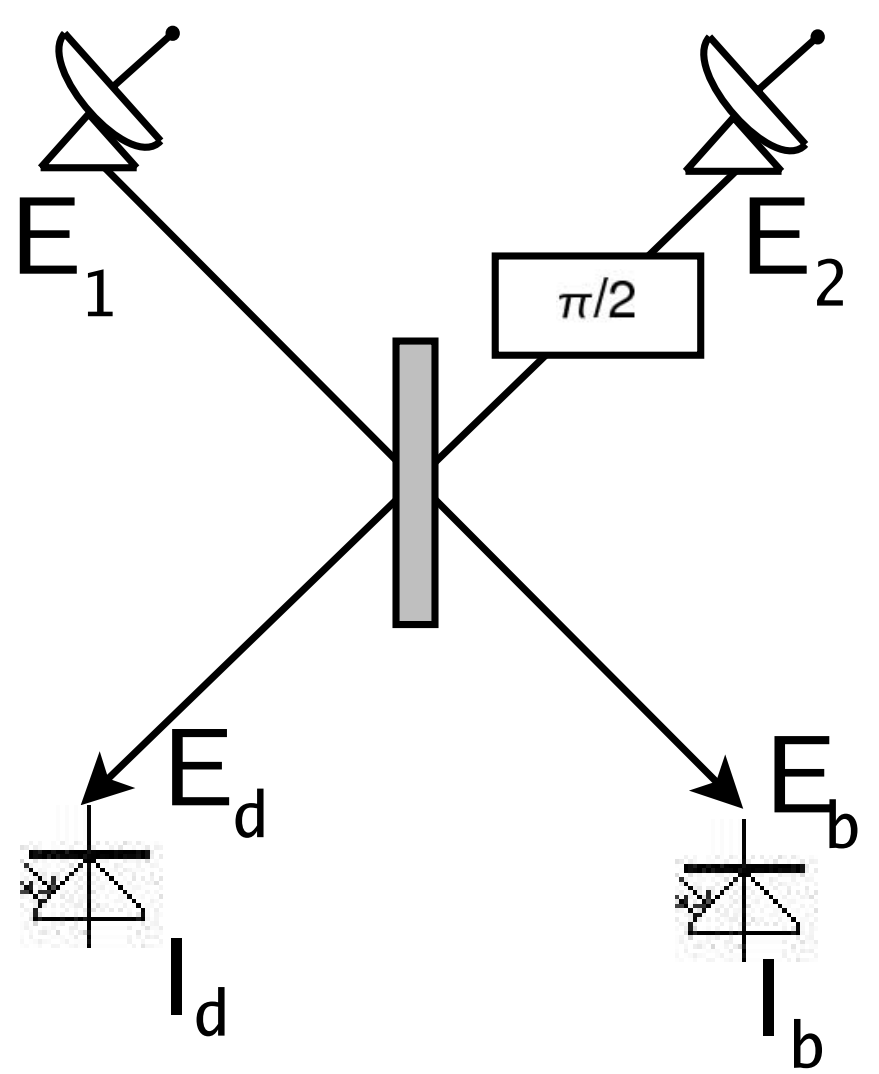

FIG. 1.-Simple beam combiner used in the Bracewell concept.

mismatches between the arms of the interferometer $(\phi \ll 1$ and $a \sim 1)$. The two output beams become

$$
\begin{aligned}
& E_{b}=\frac{E_{1}+E_{2} e^{i \pi / 2}}{\sqrt{2}}, \\
& E_{d}=\frac{E_{1} e^{i \pi / 2}+E_{2}}{\sqrt{2}},
\end{aligned}
$$

and we recover the usual interferometric fringe as

$$
\begin{aligned}
& I_{b}=\left|E_{b}\right|^{2}=\frac{A^{2}}{2}\left[a_{1}^{2}+a_{2}^{2}+2 a_{1} a_{2} \cos \left(\phi_{1}-\phi_{2}\right)\right], \\
& I_{d}=\left|E_{d}\right|^{2}=\frac{A^{2}}{2}\left[a_{1}^{2}+a_{2}^{2}-2 a_{1} a_{2} \cos \left(\phi_{1}-\phi_{2}\right)\right] .
\end{aligned}
$$

The phase difference $\phi_{1}-\phi_{2}$ has two terms: instrumental phase differences $(\delta \phi)$ and a geometric term. In the ideal planet-search situation $\delta \phi$ is small, the instrument baseline is perpendicular to the direction of the star, and the planet is at a small angle such that light from it has an additional phase given by

$$
\phi_{p}=\frac{2 \pi}{\lambda} \boldsymbol{B} \cdot \boldsymbol{s}
$$

where $\boldsymbol{B}$ is the baseline vector separating the apertures and $\boldsymbol{s}$ is the star-planet separation vector, both projected on the sky. By rotating the interferometer baseline about the direction to the star, it is possible to produce a time-variable signal in the $I_{d}$ output coming from the planet, while keeping the star nulled. If the null were ideal $\left(\delta a=a_{2}-a_{1} \sim 0\right.$ and $\left.\delta \phi \sim 0\right)$, there would be no stellar leakage to overwhelm the planet signal in the dark output $I_{d}$. Unfortunately, $I_{p} / I_{S} \sim 10^{-6}$, and hence we would require $\delta a, \delta \phi \leq 10^{-3}$.
In the absence of near-perfect amplitude and phase control, a detector at the "dark" output of the nulling interferometer will see light from two sources: the planet, which will vary according to the rotation of the interferometer, and the leakage, which may vary on all timescales. To the extent that this variability occurs at frequencies coincident with the planet signal, it will cause confusion and loss of planet signal-to-noise ratio $(\mathrm{S} / \mathrm{N})$. However, if it were possible to measure the leakage terms after the beam combination, it might be possible to significantly relax the control requirements, making the instrument more feasible. In such a situation it is no longer necessary to require that leakage levels be extremely stable; instead it is only necessary that the uncalibrated changes of the leakage (e.g., due to shot noise) be smaller than the planet signal.

One might consider monitoring the "bright" output of the nuller $I_{b}$, and use conservation of energy arguments to infer that any reduction of photon counts in $I_{b}$ implies a corresponding increase in $I_{d}$. However, for the typical TPF observation, $I_{b} \sim$ $I_{s} \sim 10^{6}$ photons $\mathrm{s}^{-1}$ and the photon noise will be too great $\left(\sqrt{I_{b}} \gg I_{p}\right)$ to determine $I_{d}$ to sufficient precision in a reasonable amount of time. This approach would also be extremely sensitive to any changes in background or detector gain, as variations at the part in $10^{6}$ level would render the calibration useless.

A different approach has also been suggested (Danchi et al. 2003), where one can take advantage of the fact that instrumental mismatches are typically in path length rather than phase, and hence will have a known wavelength dependence; this should allow one to use wide-bandwidth data to solve for and subtract systematic errors. However, such multicolor approaches are still vulnerable to, for example, polarization mismatch errors, and they will require that the nuller operate with very wide bandpasses, placing stringent requirements on the achromatic design elements.

\subsection{Calibration of a Single Bracewell Nuller}

Most of the light that leaks through the null comes from the star. Therefore, if we mix (e.g., at a beam splitter) the electric fields of the leakage with that of a separate reference beam, also from the star, fringes will form as long as the relative path delays are maintained to within the coherence length of the light (Fig. 2). On the other hand, light from the planet is not coherent with the starlight (i.e., $\left\langle E_{\text {star }} E_{\text {planet }}\right\rangle=0$, where the brackets indicate an average over time), and hence will not form fringes. If we take a portion of the two outputs (e.g., 50\%) of the nulling interferometer and recombine them again with a fourth beam splitter, the resulting electric fields of these "calibration" outputs are

$$
\begin{gathered}
E_{c, 1}=\frac{E_{b} e^{i \theta}+E_{d} e^{i \pi / 2}}{\sqrt{2}}, \\
E_{c, 2}=\frac{E_{b} e^{i(\theta+\pi / 2)}+E_{d}}{\sqrt{2}},
\end{gathered}
$$

where $\theta$ is any additional phase introduced into one of the arms of the calibration interferometer. The corresponding intensities are

$$
\begin{aligned}
I_{c, 1}(\theta)= & \frac{A^{2}}{4}\left[a_{1}^{2}+a_{2}^{2}+\left(a_{1}^{2}-a_{2}^{2}\right) \cos \left(\phi_{1}-\phi_{2}\right)\right. \\
& \left.+2 a_{1} a_{2} \sin \left(\phi_{1}-\phi_{2}\right) \sin (\theta)\right], \\
I_{c, 2}(\theta)= & \frac{A^{2}}{4}\left[a_{1}^{2}+a_{2}^{2}-\left(a_{1}^{2}-a_{2}^{2}\right) \cos \left(\phi_{1}-\phi_{2}\right)\right. \\
& \left.-2 a_{1} a_{2} \sin \left(\phi_{1}-\phi_{2}\right) \sin (\theta)\right] .
\end{aligned}
$$




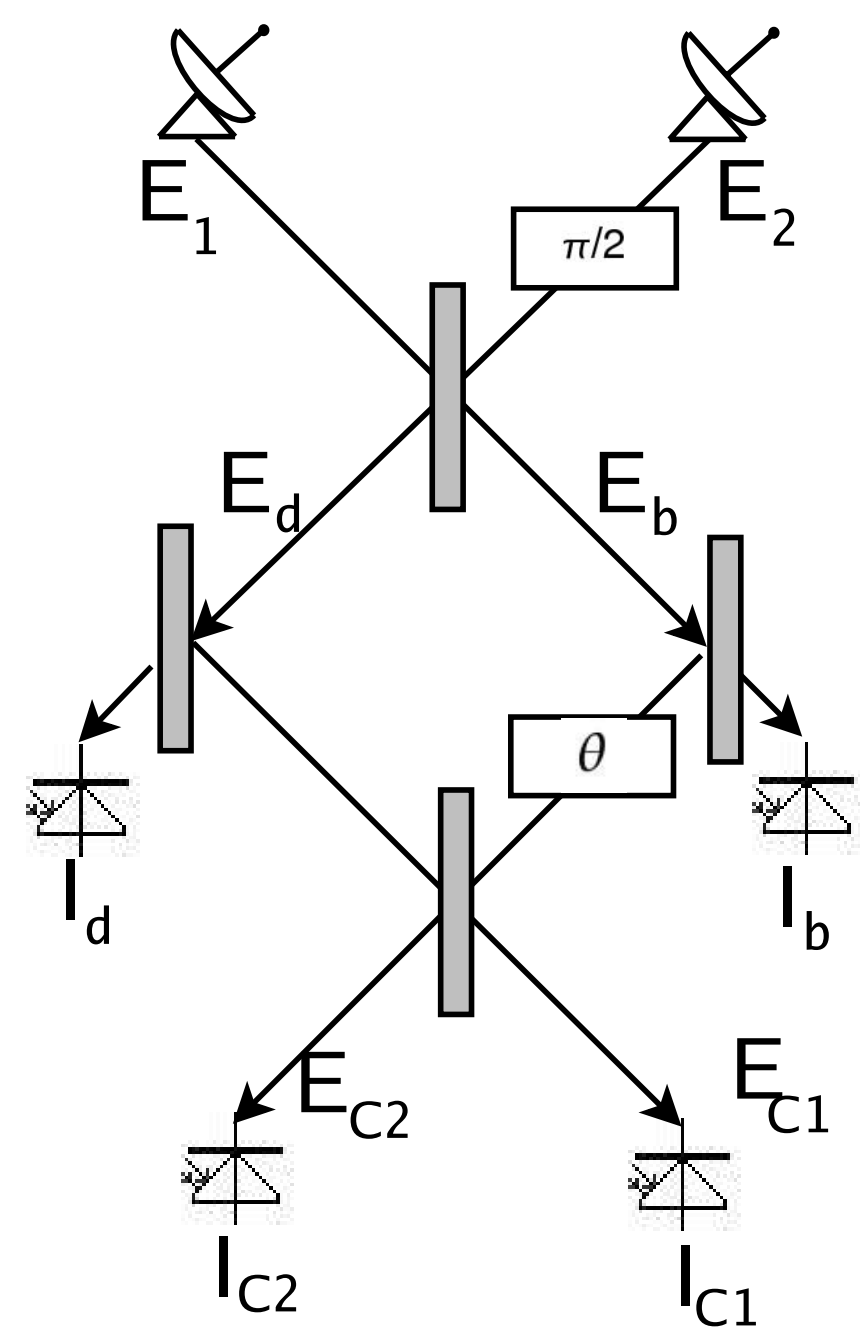

FIG. 2.-Calibration of a Bracewell null. Fractions of the bright and dark nuller outputs are recombined with a controlled relative phase shift $(\theta)$; the intensities of the calibration outputs can be used to solve for amplitude and phase mismatches in the input beams.

A fringe pattern forms in the output of the calibration interferometer; this fringe pattern contains information about the amplitude and phase mismatches of the input beams. This information can be extracted in a straightforward manner, given that $\theta$ is entirely internal to the instrument and under our control. If we measure the intensities $I_{c}$ twice, having added $\Delta \theta=\pi / 2$ to $\theta$ for the second reads, and labeling the reads " $\mathcal{A}$," " $\mathcal{B}$," " $\mathcal{C}$," and " $\mathcal{D}$;" i.e.,

$$
\begin{gathered}
\mathcal{A}=I_{c, 1}(\theta=0), \\
\mathcal{B}=I_{c, 2}(\theta=0), \\
\mathcal{C}=I_{c, 1}(\theta=\pi / 2), \\
\mathcal{D}=I_{c, 2}(\theta=\pi / 2),
\end{gathered}
$$

these measurements can be used to "reconstruct" the output $I_{d}$ caused by amplitude and phase mismatches, i.e., the leakage through the null. Then

$$
\tilde{I}_{d}=\frac{(\mathcal{A}-\mathcal{B})^{2}+(\mathcal{C}-\mathcal{D})^{2}}{4 I_{b}} .
$$

This leakage can be subtracted from the measured $I_{d}$; the remaining light is from the planet. While it is true that the planet light will also pass through the calibration interferometer and create an interference fringe- - because the light from the planet is not coherent with the starlight, yet both fall on the same detector - the fringe contrast of the fringe due to the planet will be reduced by a factor of $I_{p} / I_{s} \sim 10^{-6}$ and will have a negligible impact on the measurement of the leakage parameters.

A nulling interferometer will have starlight leak through the null for two reasons: the first is due to amplitude and path (and polarization) mismatches, as discussed above. The second is simply due to the finite size of the star. Even a perfect null only blocks light that is exactly on-axis; for typical stellar sizes and distances the star will subtend an apparent angle on the order of a milliarcsecond, large enough that a nonnegligible amount of light will leak through the null. This leakage cannot be removed by the proposed calibration technique. However, the level of this leakage is set by the length of the nulling baseline and should not vary on timescales comparable to the planet signal. Nonetheless, shot noise from the light that leaks through can overwhelm the planet signal; it is this leakage term that limits the maximum size of the nulling baselines.

It is interesting to note that the reconstructed leakage through the null has more favorable noise properties than a leakage term reconstructed from the bright output alone; this is a manifestation of "heterodyne gain." Assuming that the calibration intensity measurements are dominated by photon noise (i.e., $\sigma_{\mathcal{A}}^{2}=\mathcal{A}$ ) the uncertainty of the calibration measurement is given by

$$
\sigma_{\tilde{I}_{d}}^{2}=\sum_{R_{i}=I_{b}, \mathcal{A} \ldots \mathcal{D}}\left|\frac{\partial \tilde{I}_{d}}{\partial R_{i}}\right|^{2} \sigma_{R_{i}}^{2}=\sigma_{I_{d}}^{2} \approx \frac{A^{2}\left(\delta a^{2}+\delta \phi^{2}\right)}{4} .
$$

In other words, the reconstructed leakage $\tilde{I}_{d}$ has the same $\mathrm{S} / \mathrm{N}$ as what one would find by measuring $I_{d}$ itself at the dark output, except that now there is negligible contamination due to light from the planet; the mixing process allows one to separate the two cleanly.

For $\delta a \sim 10^{-3}, \delta \phi \sim 10^{-3}, A^{2}=\tau I_{s}$, and $I_{s} \sim 10^{6}$ photons s ${ }^{-1}$, assuming $\tau \sim 100 \mathrm{~s}$, one should expect to measure $\tilde{I}_{d}$ to a precision of $\sim 4 \times 10^{-3}$. The price for this calibration is a loss in the effective throughput of the instrument, as half of the light from the planet gets lost in the calibration system. Clearly there is a trade-off between the need to minimize photon noise from the planet versus noise due to time-variable null leakage; we discuss the effect this has on the overall instrument design in $\S 4$.

\subsection{Simulations of a Calibrated Single Bracewell Nuller}

We have simulated the operation of a simple two-aperture Bracewell interferometer observing an Earth-like planet. We assumed a baseline of $50 \mathrm{~m}, 5.5 \mathrm{~m}$ diameter collecting apertures, and an Earth-sized planet in a 1 AU orbit with an albedo of 0.3 . The central star was modeled as a $5700 \mathrm{~K}, 1 R_{\odot}$ blackbody located at a distance of $10 \mathrm{pc}$. Total integration time is $2.16 \times 10^{5} \mathrm{~s}$, spread out over two full $360^{\circ}$ rotations of the array, with $300 \mathrm{~s}$ of integration time at each orientation. Path-length errors for each integration were modeled as $10 \mathrm{~nm}$ rms Gaussian random noise, and amplitude mismatch errors had a fractional amplitude of 0.001 . Photon noise was applied to all measured intensities. The system efficiency was assumed to be $30 \%$. We assumed the system to have five $1 \mu \mathrm{m}$ wide spectral channels ranging from 8 to $12 \mu \mathrm{m}$ in central wavelength.

It is clear from Figure 3 that the leakage due to amplitude and phase mismatches completely overwhelms the planet signal, while the calibration successfully removed the leakage and recovered the planet. However, the required integration time and aperture size was considerable - a consequence of the single-Bracewell 

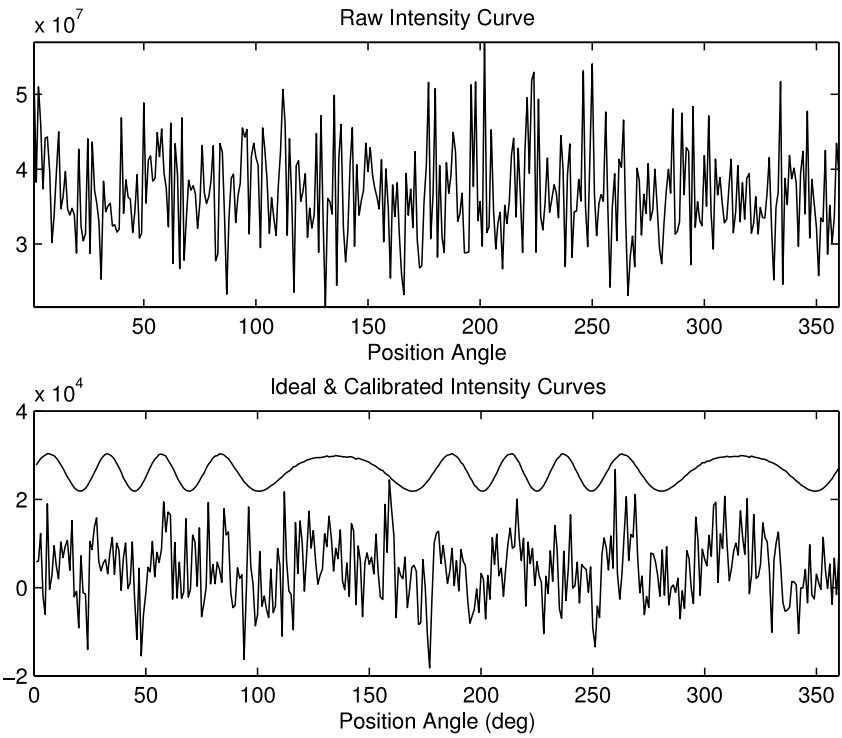

FIG. 3.-Top: Raw intensities produced by the simulated two-aperture Bracewell interferometer, in a single wavelength channel, as a function of array rotation angle. Bottom: Calibrated output, together with the ideal time series that would be expected from an interferometer with no noise. The ideal curve has been offset for clarity. Note that calibration has removed noise levels that were a factor of $10^{3}$ times greater than the planet signal.

design that is limited by the trade-off between null depth and angular resolution. With only two apertures, the finite size of the stellar disk will cause light to leak through the null if the baseline is long enough to resolve the planet. Nevertheless, we reconstruct images of the target system using the standard cross-correlation analysis described by Lay (2005). The simulated light curves (both raw and calibrated) are cross-correlated with a series of templates. The templates are a function of the position of a planet and are given by the theoretical response of a two-aperture interferometer, i.e., equation (5). The results are shown in Figure 4.

\section{THE DUAL BRACEWELL NULLER}

$T P F$ has been envisioned not as a simple two-aperture Bracewell design, but rather a somewhat more sophisticated dual-Bracewell design. This is for two reasons: the response function of the twoaperture Bracewell is symmetric, leading to a $180^{\circ}$ ambiguity in the position angle of any planet (Fig. 4), and in the two-aperture Bracewell the planet signal can only be modulated on the arrayrotation timescale. That timescale is $\sim 1000 \mathrm{~s}$, long enough to be susceptible to systematic errors due to instrumental drifts.

A solution to both of these problems is achieved in the form of the "dual chopped Bracewell" design outlined in Figure 5. In this configuration two Bracewell pairs are placed next to each other, and the nulled outputs from each are recombined with relative phase shifts that are switched between values 0 and $\pi$. Such phase chopping produces an asymmetric response on the sky, and since the internal phase shift can be adjusted at high frequency, it allows one to remove many forms of systematic error associated with the instrument gain and background.

We derive the output of a dual Bracewell system below:

$$
\begin{gathered}
E_{1}=A a_{1} e^{i\left(\phi_{1}\right)}, \\
E_{2}=A a_{2} e^{i\left(\phi_{2}+\pi / 2\right)}, \\
E_{3}=A a_{3} e^{i\left(\phi_{3}\right)}, \\
E_{4}=A a_{4} e^{i\left(\phi_{4}+\pi / 2\right)} .
\end{gathered}
$$
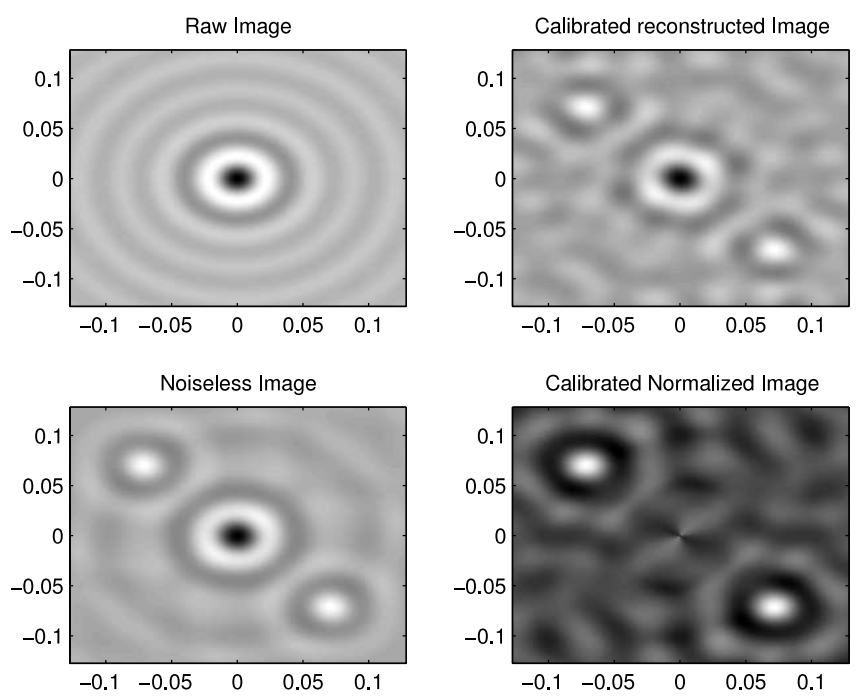

FIG. 4.-Top left: Reconstructed image using simulated uncalibrated multichannel data from a single Bracewell nuller. Top right: Reconstructed image of the same system, now using the calibrated data. Bottom left: The image that would be expected from an ideal (noiseless) system. Bottom right: Reconstructed calibrated image, with the central point-spread function feature (due to leakage through the null) divided out, using a rotationally averaged raw image as the template. Note the $180^{\circ}$ position ambiguity inherent in two-aperture instruments.

The apertures are combined pairwise to create "left" and "right" combinations as follows:

$$
\begin{aligned}
& E_{d, L}=\frac{E_{1}+E_{2} e^{i \pi / 2}}{\sqrt{2}}, \\
& E_{b, L}=\frac{E_{1} e^{i \pi / 2}+E_{2}}{\sqrt{2}}, \\
& E_{d, R}=\frac{E_{3}+E_{4} e^{i \pi / 2}}{\sqrt{2}}, \\
& E_{b, R}=\frac{E_{3} e^{i \pi / 2}+E_{4}}{\sqrt{2}} .
\end{aligned}
$$

Combining the two nulled outputs with relative phases $0, \pi$, which we label the "A" and "B" states, respectively, yields

$$
\begin{aligned}
E_{d d 1, A} & =\frac{E_{d, L}+E_{d, R} e^{i \pi / 2}}{\sqrt{2}}, \\
E_{d d 1, B} & =\frac{E_{d, L}+E_{d, R} e^{i 3 \pi / 2}}{\sqrt{2}},
\end{aligned}
$$

and the corresponding intensities are

$$
\begin{aligned}
I_{d d 1, A}= & \frac{A^{2}}{4}\left[a_{1}^{2}+a_{2}^{2}+a_{3}^{2}+a_{4}^{2}-2 a_{1} a_{2} \cos \left(\phi_{1}-\phi_{2}\right)\right. \\
& +2 a_{1} a_{3} \sin \left(\phi_{1}-\phi_{3}\right)-2 a_{1} a_{4} \sin \left(\phi_{1}-\phi_{4}\right) \\
& -2 a_{2} a_{3} \sin \left(\phi_{2}-\phi_{3}\right)+2 a_{2} a_{4} \sin \left(\phi_{2}-\phi_{4}\right) \\
& \left.-2 a_{3} a_{4} \cos \left(\phi_{3}-\phi_{4}\right)\right], \\
I_{d d 1, B}= & \frac{A^{2}}{4}\left[a_{1}^{2}+a_{2}^{2}+a_{3}^{2}+a_{4}^{2}-2 a_{1} a_{2} \cos \left(\phi_{1}-\phi_{2}\right)\right. \\
& -2 a_{1} a_{3} \sin \left(\phi_{1}-\phi_{3}\right)+2 a_{1} a_{4} \sin \left(\phi_{1}-\phi_{4}\right) \\
& +2 a_{2} a_{3} \sin \left(\phi_{2}-\phi_{3}\right)-2 a_{2} a_{4} \sin \left(\phi_{2}-\phi_{4}\right) \\
& \left.-2 a_{3} a_{4} \cos \left(\phi_{3}-\phi_{4}\right)\right] .
\end{aligned}
$$




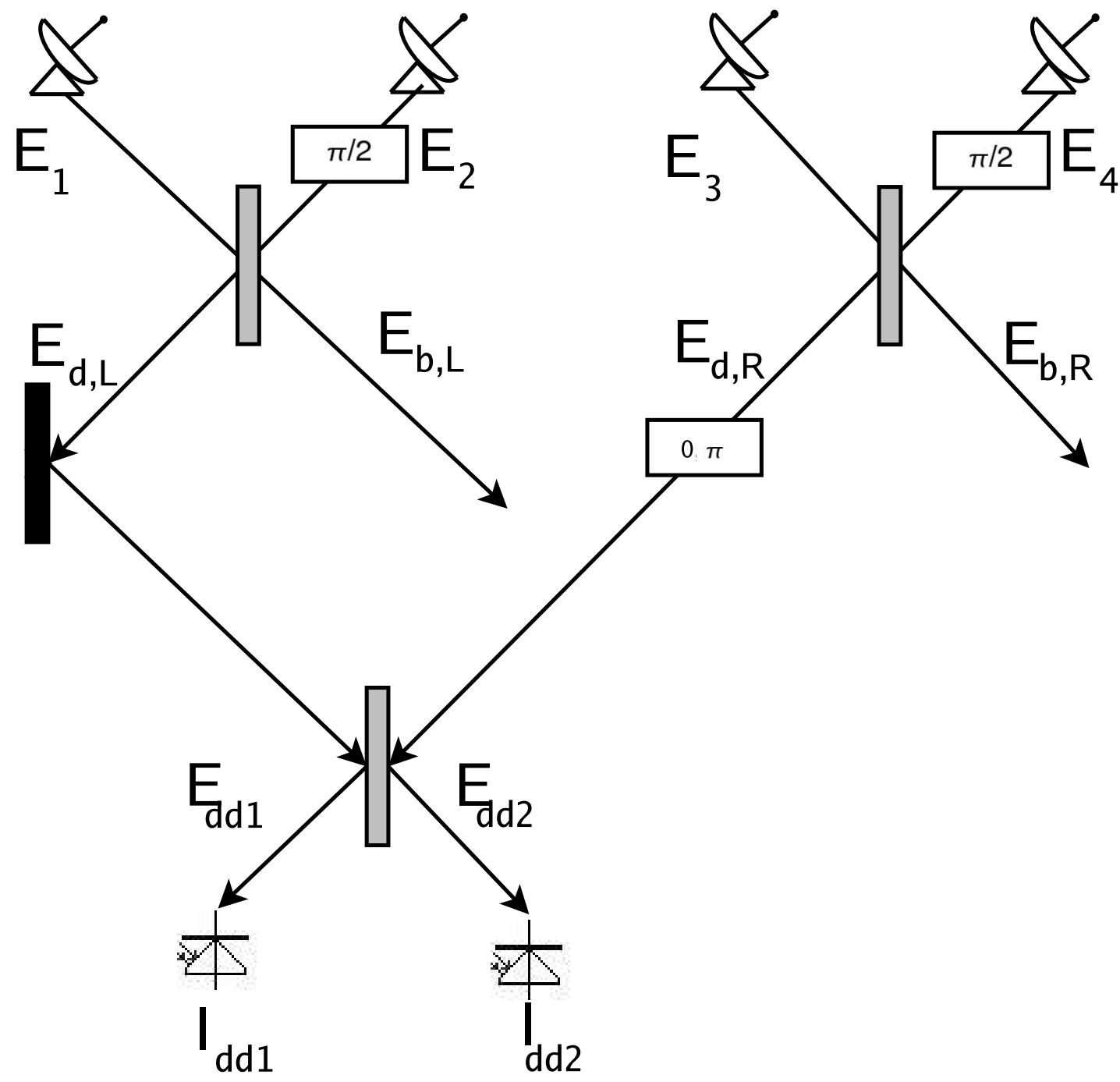

FIG. 5.-Schematic layout of the dual, chopped Bracewell configuration. The two aperture pairs 1,2 and 3, 4 are combined so as to create nulls; the nulled outputs are then cross-combined. A relative phase of 0 or $\pi$ is imposed between combinations.

The final quantities used in the image reconstruction are the "sine" and "cosine" chops. The sine chop is given by

$$
\begin{aligned}
I_{\mathrm{sin}}= & I_{d d 1, A}-I_{d d 1, B} \\
= & A^{2}\left[a_{1} a_{3} \sin \left(\phi_{1}-\phi_{3}\right)-a_{2} a_{3} \sin \left(\phi_{2}-\phi_{3}\right)\right. \\
& \left.-a_{1} a_{4} \sin \left(\phi_{1}-\phi_{4}\right)+a_{2} a_{4} \sin \left(\phi_{2}-\phi_{4}\right)\right] .
\end{aligned}
$$

This gives a response that is asymmetric with respect to the phase center of the instrument, and hence will allow one to determine the position angle of any planet without the $180^{\circ}$ ambiguity of the single-Bracewell configuration. However, note that in this case, small amplitude and phase mismatches can couple, as the sine chop now contains error terms of the form $\delta a \delta \phi$. A particularly challenging aspect of this coupling is that there are higher order terms that couple nonlinearly. Given that these mismatches occur on a range of timescales, the nonlinear mixing can inject noise at frequencies where the planet signal is maximized, even in the presence of servo-control systems that try to minimize the mismatches.

$$
\begin{aligned}
& \text { The "cosine chop" is } \\
& \begin{aligned}
I_{\mathrm{cos}}= & I_{d d 1, A}+I_{d d 1, B} \\
= & \frac{A^{2}}{2}\left[a_{1}^{2}+a_{2}^{2}+a_{3}^{2}+a_{4}^{2}-2 a_{1} a_{2} \cos \left(\phi_{1}-\phi_{2}\right)\right. \\
& \left.\quad-2 a_{3} a_{4} \cos \left(\phi_{3}-\phi_{4}\right)\right] .
\end{aligned}
\end{aligned}
$$

In general the sine chop is more useful to the image reconstruction, as the cosine chop will include contributions from symmetric sources such as zodiacal light. In any case, if the instrument is switched between states A and B quickly enough to remove drifts in thermal background, gain, etc., then these quantities are primarily determined by the planet. However, there are still the effects of variable leakage to consider and avoid.

\subsection{Calibration of a Dual Bracewell Nuller}

The concept of coherent calibration using the nonnulled outputs from the nulling interferometers can be applied in a straightforward manner to the dual Bracewell configuration (Fig. 6). In this configuration, the bright (nonnulled) outputs from the pairwise 


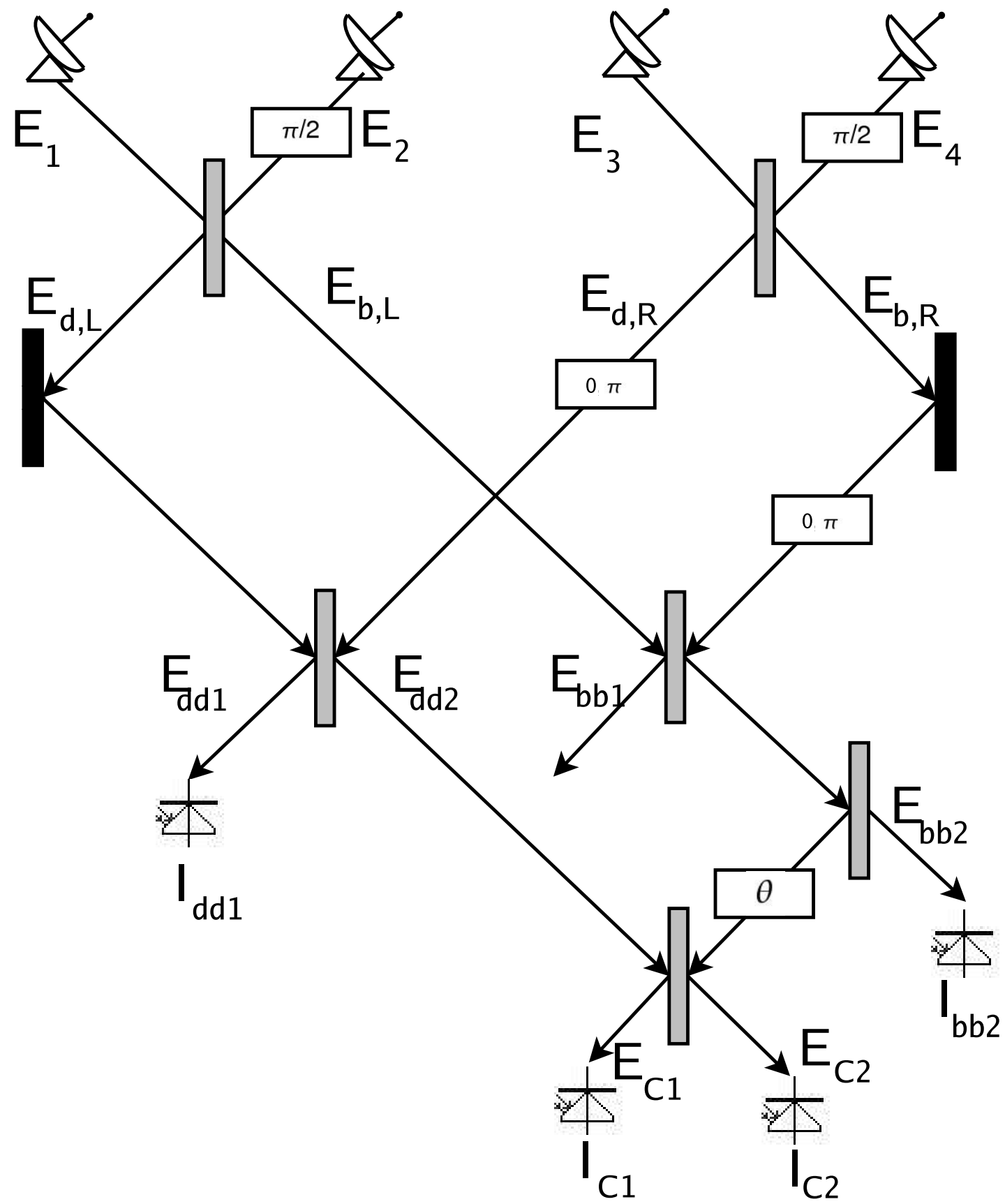

FIG. 6.-Schematic layout of the dual, chopped Bracewell configuration with calibration.

nullers are combined, and this beam is then mixed with the combined, nulled output. The resulting electric fields and intensities are easily found:

$$
\begin{gathered}
E_{c 1}=\frac{E_{d d, 2}+E_{b b, 2} e^{i(\theta+\pi / 2)} / \sqrt{2}}{\sqrt{2}}, \\
E_{c 2}=\frac{E_{d d, 2} e^{i \pi / 2}+E_{b b, 2} e^{i \theta} / \sqrt{2}}{\sqrt{2}},
\end{gathered}
$$

where $\theta$ is an additional, controllable phase shift that can be introduced between the bright and dark beams. The associated intensities are, after some algebra, found as

$$
\begin{aligned}
I_{c 1, A}= & \frac{A^{2}}{16}\left\{3\left(a_{1}^{2}+a_{2}^{2}+a_{3}^{2}+a_{4}^{2}\right)\right. \\
& +2 \sqrt{2} \cos (\theta)\left(-a_{1}^{2}+a_{2}^{2}-a_{3}^{2}+a_{4}^{2}\right) \\
& +4 \sqrt{2} \cos (\theta)\left[a_{1} a_{3} \sin \left(\phi_{1}-\phi_{3}\right)-a_{2} a_{4} \sin \left(\phi_{2}-\phi_{4}\right)\right] \\
& +4 \sqrt{2} \sin (\theta)\left[-a_{1} a_{2} \sin \left(\phi_{1}-\phi_{2}\right)-a_{1} a_{4} \cos \left(\phi_{1}-\phi_{4}\right)\right. \\
& \left.-a_{2} a_{3} \cos \left(\phi_{2}-\phi_{3}\right)+a_{3} a_{4} \sin \left(\phi_{3}-\phi_{4}\right)\right] \\
& -2 a_{1} a_{2} \cos \left(\phi_{1}-\phi_{2}\right)-6 a_{1} a_{3} \sin \left(\phi_{1}-\phi_{3}\right) \\
& +2 a_{1} a_{4} \sin \left(\phi_{1}-\phi_{4}\right)+2 a_{2} a_{3} \sin \left(\phi_{2}-\phi_{3}\right) \\
& \left.-6 a_{2} a_{4} \sin \left(\phi_{2}-\phi_{4}\right)-2 a_{3} a_{4} \cos \left(\phi_{3}-\phi_{4}\right)\right\},
\end{aligned}
$$



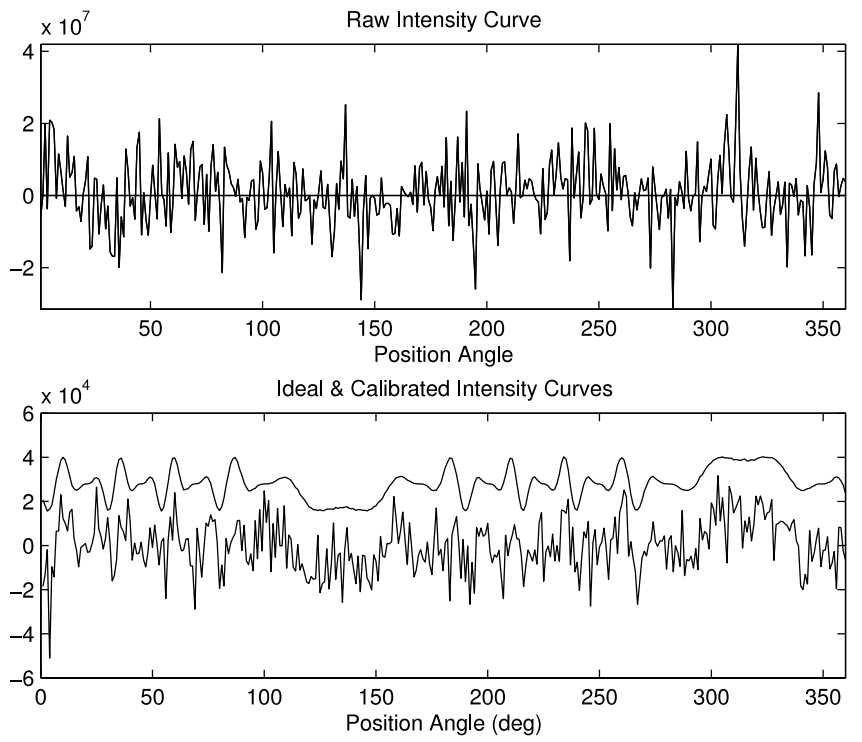

FIG. 7.-Top: Intensities produced by the simulated four-aperture, dual chopped Bracewell interferometer, for a single spectral channel, as a function of array rotation angle. "Raw" is the intensity $I_{\text {sin }}$ including simulated planet and noise sources as explained in the text. Bottom: Calibrated output, together with the ideal time series that would be expected from an interferometer with no phase noise. The ideal curve has been offset for clarity.

$$
\begin{aligned}
I_{c 2, A}= & \frac{A^{2}}{16}\left\{3\left(a_{1}^{2}+a_{2}^{2}+a_{3}^{2}+a_{4}^{2}\right)\right. \\
& +2 \sqrt{2} \cos (\theta)\left(a_{1}^{2}-a_{2}^{2}+a_{3}^{2}-a_{4}^{2}\right) \\
& +4 \sqrt{2} \cos (\theta)\left[-a_{1} a_{3} \sin \left(\phi_{1}-\phi_{3}\right)+a_{2} a_{4} \sin \left(\phi_{2}-\phi_{4}\right)\right] \\
& +4 \sqrt{2} \sin (\theta)\left[a_{1} a_{2} \sin \left(\phi_{1}-\phi_{2}\right)+a_{1} a_{4} \cos \left(\phi_{1}-\phi_{4}\right)\right. \\
& \left.-a_{2} a_{3} \cos \left(\phi_{2}-\phi_{3}\right)+a_{3} a_{4} \sin \left(\phi_{3}-\phi_{4}\right)\right] \\
& -2 a_{1} a_{2} \cos \left(\phi_{1}-\phi_{2}\right)-6 a_{1} a_{3} \sin \left(\phi_{1}-\phi_{3}\right) \\
& +2 a_{1} a_{4} \sin \left(\phi_{1}-\phi_{4}\right)+2 a_{2} a_{3} \sin \left(\phi_{2}-\phi_{3}\right) \\
& \left.-6 a_{2} a_{4} \sin \left(\phi_{2}-\phi_{4}\right)-2 a_{3} a_{4} \cos \left(\phi_{3}-\phi_{4}\right)\right\}, \\
I_{c 1, B}= & \frac{A^{2}}{16}\left\{3\left(a_{1}^{2}+a_{2}^{2}+a_{3}^{2}+a_{4}^{2}\right)\right. \\
& +2 \sqrt{2} \cos (\theta)\left(-a_{1}^{2}+a_{2}^{2}-a_{3}^{2}+a_{4}^{2}\right) \\
& +4 \sqrt{2} \cos (\theta)\left[-a_{1} a_{3} \sin \left(\phi_{1}-\phi_{3}\right)+a_{2} a_{4} \sin \left(\phi_{2}-\phi_{4}\right)\right] \\
& +4 \sqrt{2} \sin (\theta)\left[-a_{1} a_{2} \sin \left(\phi_{1}-\phi_{2}\right)+a_{1} a_{4} \cos \left(\phi_{1}-\phi_{4}\right)\right. \\
& \left.-a_{2} a_{3} \cos \left(\phi_{2}-\phi_{3}\right)-a_{3} a_{4} \sin \left(\phi_{3}-\phi_{4}\right)\right] \\
& -2 a_{1} a_{2} \cos \left(\phi_{1}-\phi_{2}\right)+6 a_{1} a_{3} \sin \left(\phi_{1}-\phi_{3}\right) \\
& -2 a_{1} a_{4} \sin \left(\phi_{1}-\phi_{4}\right)-2 a_{2} a_{3} \sin \left(\phi_{2}-\phi_{3}\right) \\
& \left.+6 a_{2} a_{4} \sin \left(\phi_{2}-\phi_{4}\right)-2 a_{3} a_{4} \cos \left(\phi_{3}-\phi_{4}\right)\right\}, \\
& A^{2}\left\{3\left(a_{1}^{2}+a_{2}^{2}+a_{3}^{2}+a_{4}^{2}\right)\right. \\
& \left.+6 a_{2} a_{4} \sin \left(\phi_{2}-\phi_{4}\right)-2 a_{3} a_{4} \cos \left(\phi_{3}-\phi_{4}\right)\right\} . \\
& +2 \sqrt{2} \cos (\theta)\left(a_{1}^{2}-a_{2}^{2}+a_{3}^{2}-a_{4}^{2}\right) \\
& +4 \sqrt{2} \cos (\theta)\left[a_{1} a_{3} \sin \left(\phi_{1}-\phi_{3}\right)-a_{2} a_{4} \sin \left(\phi_{2}-\phi_{4}\right)\right] \\
& +4 \sqrt{2} \sin (\theta)\left[a_{1} a_{2} \sin \left(\phi_{1}-\phi_{2}\right)-a_{1} a_{4} \cos \left(\phi_{1}-\phi_{4}\right)\right. \\
& \left.+a_{2} a_{3} \cos \left(\phi_{2}-\phi_{3}\right)+a_{3} a_{4} \sin \left(\phi_{3}-\phi_{4}\right)\right] \\
& -2 a_{1} a_{2} \cos \left(\phi_{1}-\phi_{2}\right)+6 a_{1} a_{3} \sin \left(\phi_{1}-\phi_{3}\right) \\
& -2 a_{1} a_{4} \sin \left(\phi_{1}-\phi_{4}\right)-2 a_{2} a_{3} \sin \left(\phi_{2}-\phi_{3}\right) \\
& +210
\end{aligned}
$$
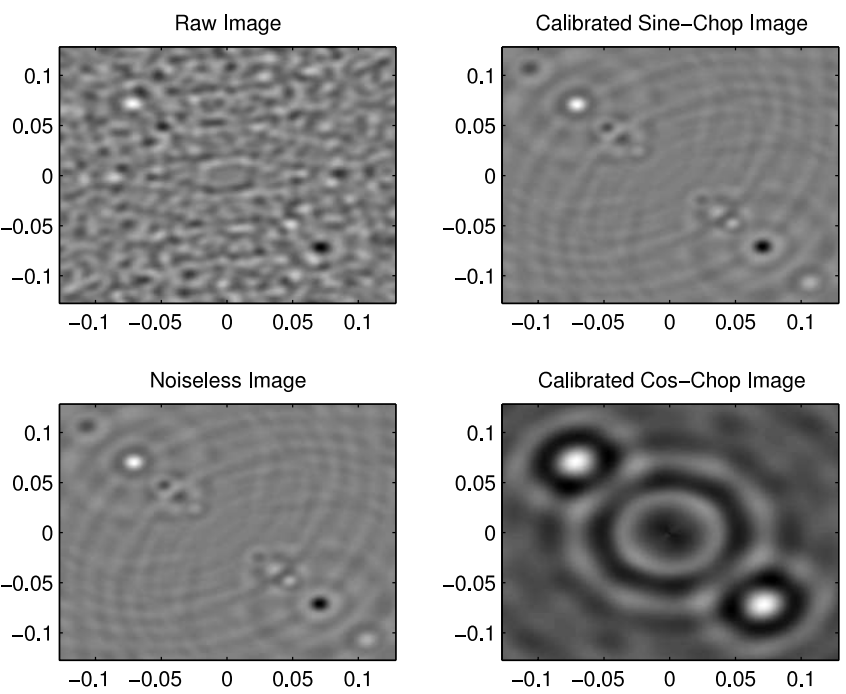

FIG. 8.-Top left: Reconstructed image using simulated uncalibrated data from a dual Bracewell nuller. Top right: Reconstructed image of the same system, now using the calibrated data. Bottom left: Image that would be expected from an ideal (noiseless) system. Bottom right: Image reconstructed from the calibrated cosine chop. The $180^{\circ}$ position ambiguity disappears for the sine-chop data, as the dual Bracewell nuller provides the necessary phase information to determine the position of the planet. Also note that the sine chop includes terms that depend on the phase difference between apertures 1-3, 2-4, and 1-4, with longer effective baselines; hence the reconstructed image shows much higher spatial resolution. Note that there is only one planet in the simulation; the dirty map produced by the cross-correlation does include artifacts. A proper deconvolution, e.g., based on the CLEAN algorithm (Draper et al. 2006), is beyond the scope of this paper.

If we measure the outputs $I_{c 1}, I_{c 2}$ with $\theta=0$ and $\pi / 2$, in the fashion analogous to what is done for the single-Bracewell case (i.e., eqs. [12] and [16]), we can reconstruct the output from $I_{d d 1}$; this works for both chop states. Hence we can recover $I_{\sin }$ and $I_{\cos }$.

The layout shown in Figure 6 is not the only one possible. In fact, one could use any bright output as an input to the calibrator, including the outputs from just a single nuller $\left(E_{b, L}\right.$ or $\left.E_{b, R}\right)$. As discussed in $\S 5.4$, there are some advantages to this particular layout, in that it can be made fully symmetric.

\subsection{Simulations of a Calibrated Dual Bracewell Nuller}

We have simulated the operation of a standard four-aperture dual-Bracewell interferometer observing an Earth-like planet (Figs. 7 and 8). Parameters were identical to the single-Bracewell case where applicable; the array was modeled as a linear fourelement array with a $50 \mathrm{~m}$ baseline. The integration time was split equally between the chopped states A and B. As in the singleBracewell case, images were reconstructed using cross-correlations of expected signals as a function of planet position (eqs. [30] and [31]).

The gain from application of the coherent calibration approach becomes clear in Figure 9. Here we create model images for three cases of noise: 1,10 , and $50 \mathrm{~nm}$ of phase and $0.01 \%, 0.1 \%$, and $0.5 \%$ of amplitude mismatch respectively. The calibration easily recovers the planet in all cases, whereas for the raw data only the lowest noise case yields a planet detection in the image.

\section{IMPLICATIONS FOR TPF INSTRUMENT DESIGN}

Using coherent calibration it is possible to measure starlight leakage through the null due to systematic path and amplitude mismatches to high precision. In the case in which such leakage is the dominant noise source, this allows for a considerable relaxation in the associated path length and amplitude control 


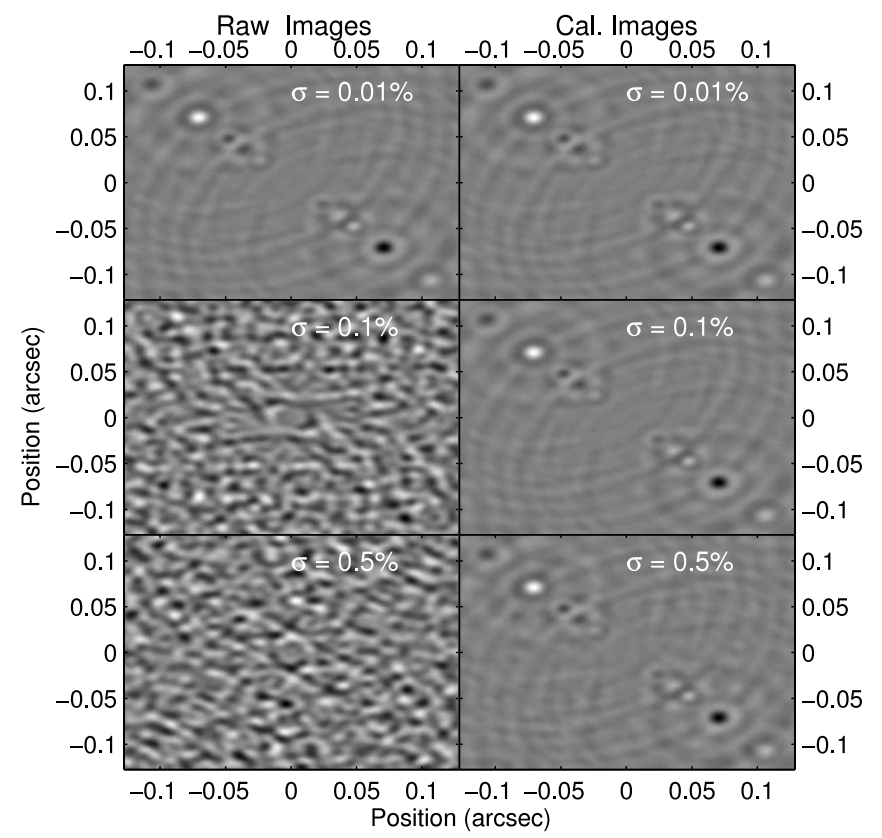

FIG. 9.- Simulated images reconstructed from raw and calibrated data, with three different noise levels. Top: $1 \mathrm{~nm}$ of phase noise and $0.01 \%$ amplitude mismatch. Middle: $10 \mathrm{~nm}$ of phase noise and $0.1 \%$ amplitude mismatch. Bottom: $50 \mathrm{~nm}$ of phase noise and $0.5 \%$ amplitude mismatch.

requirements. However, there are many sources of noise in the $T P F$ instrument, and not all of them are amenable to this type of calibration (Fig. 10). For instance, local zodiacal light produces large fluxes on the detector at longer wavelengths; Poisson fluctuations in that flux can wash out the signal from a planet.

Given that any TPF design must carefully weigh the relative contributions for many noise sources, the ability to calibrate and

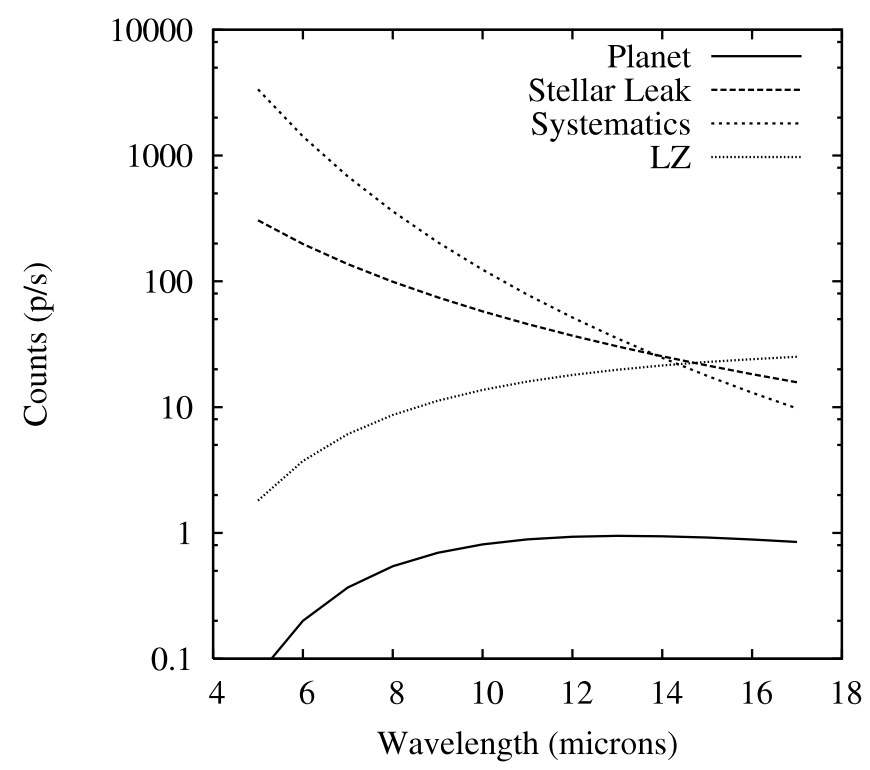

FIG. 10.- Selected noise terms in the $T P F-I$ signal-to-noise budget as a function of wavelength. Terms include local zodiacal emission, starlight leakage due to the finite size of the central star, and "systematic" leakage terms due to small path length and amplitude mismatches. We emphasize that these calculations are merely approximate; a full, high-fidelity $\mathrm{S} / \mathrm{N}$ budget for a nulling interferometer is beyond the scope of this paper. Nonetheless, it should serve to illustrate the point that - as is appropriate in any well-optimized instrument system - there are multiple competing noise sources of similar magnitude.

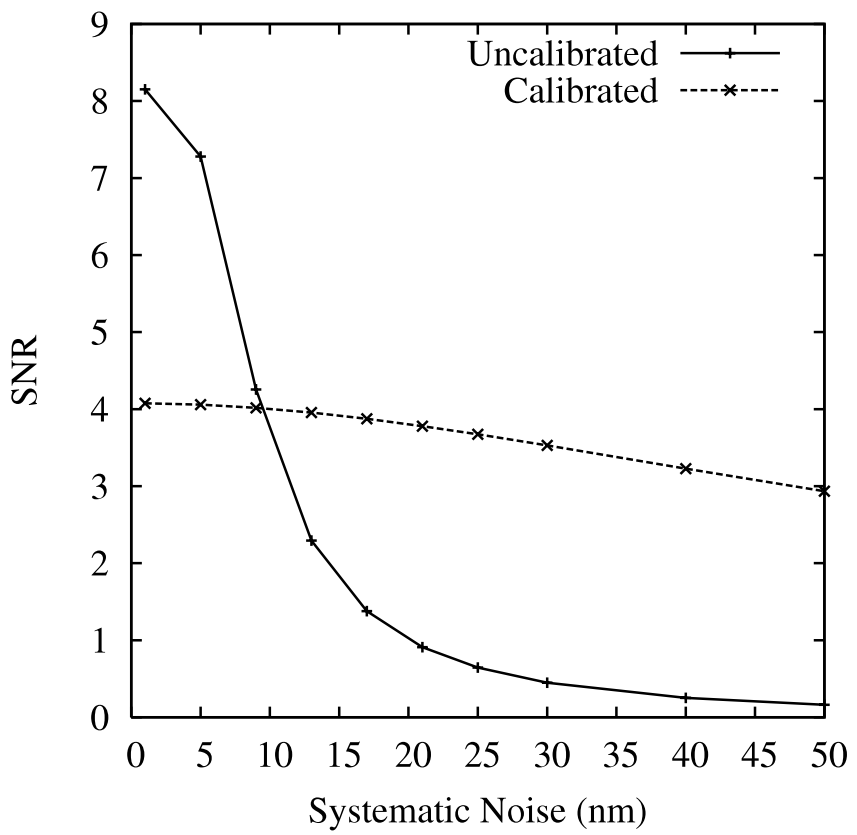

FIG. 11.- S/N of an Earth-like planet seen by a $T P F$ interferometer, for various levels of systematic noise, with and without coherent calibration. The calibration sacrifices photons in return for a much greater tolerance against systematic errors.

remove a large set of noise terms will undoubtedly influence what the optimal system design will be. A full investigation of such a system optimization is beyond the scope of this paper. However, given that the effects of small errors in phase and amplitude mismatch are significantly worse at shorter wavelengths, e.g., $\propto \lambda^{-3}$ (Lay 2005), it is likely that a TPF-like instrument equipped with a nulling calibration system will be able to work at shorter wavelengths, something that may be particularly valuable given the presence of spectral features associated with $\mathrm{H}_{2} \mathrm{O}$ around $6.3 \mu \mathrm{m}$ and $\mathrm{CH}_{4}$ at $7.7 \mu \mathrm{m}$ (Des Marais et al. 2002). Unfortunately, the underlying $\sim 300 \mathrm{~K}$ planetary blackbody emission is dropping rapidly shortward of $10 \mu \mathrm{m}$, making observations in that wavelength range very difficult, coherent calibration notwithstanding.

Perhaps the clearest illustration of the effect of coherent calibration is apparent in Figure 11, which shows the $\mathrm{S} / \mathrm{N}$ values for calibrated and uncalibrated versions of a notional TPF configuration, as a function of the level of path length stability achieved. Note that we considered only the amplitude-phase cross term in this calculation; there are additional terms that a real instrument would have to consider (e.g., related to polarization). Clearly, if $\sim 1 \mathrm{~nm}$ levels are possible, coherent calibration may not be worthwhile. However, this represents a fractional stability approaching 1 part in $10^{4}$, which may be impossible to achieve in practice, in which case coherent calibration is very useful.

\section{LIMITATIONS AND ALTERNATIVE APPROACHES}

There are a number of practical considerations and challenges to implementing this concept in a working interferometer.

\subsection{Low Fringe Visibility}

The leakage calibration quantities are extracted from a fringe in the calibration interferometer. For a deep null $\left(I_{b} / I_{d} \sim 10^{6}\right.$ or more) this implies a very low fringe contrast or visibility $\left[\sim\left(I_{b} / I_{d}\right)^{1 / 2}\right]$; such low fringe visibilities may be hard to measure in the presence of certain kinds of systematic noise (e.g., detector noise) that can produce an additive bias to the measured 
fringe contrast. However, such additive biases can be measured and removed - and they are most easily measured with a brighter source. It is therefore always preferable to have the brightest possible reference beam (as long as the detector is not saturated), even if the $\mathrm{S} / \mathrm{N}$ of the leakage measurement is to first order independent of the brightness of the "bright" input to the calibration interferometer.

\subsection{Thermal Noise}

While thermal foreground emission (e.g., from the telescope optics) is usually considered incoherent in that it does not form fringes at the nuller, this is not the case for a calibrator. The calibration interferometer in effect forms a Mach-Zender interferometer in the instrument, and hence any light that enters one input port will form a fringe in the calibrator. We note that this fringe can be measured and corrected simply by blocking one input of the nuller and measuring the amplitude of a remaining fringe. It should also be noted that for all but the longest wavelengths the amount of thermal emission that enters the system and forms a fringe in the calibrator will be substantially smaller than the flux from a planet.

\subsection{Splitting Ratio}

For the purposes of this paper, we have assumed that half of the "dark" output is split off and mixed in the calibration interferometer, with the associated loss of S/N. However, the optimal choice of splitting ratio is probably not 50/50 and may in fact be much smaller. Since the frequency at which the planet signal varies is on the order of the array rotation timescale, it may be sufficient to only split off enough light to measure changes in the nuller leakage on that much longer timescale.

\subsection{Non-single-mode Effects}

In this discussion we have treated the incoming electric fields as single-mode wave fronts. Such an approach is appropriate for initial explorations of the concept; it is also a reasonably good approximation in the case of a space-borne instrument equipped with single-mode filters of the type envisioned for TPF (Lay 2005). However, care may be necessary in designing the instrument so as to ensure that the calibration system samples the exact same spatial mode as the science detector. In practice there may be small alignment differences that cannot easily be removed. However, we suggest that these differences can be calibrated as follows. If the layout shown in Figure 6 is made fully symmetric by adding a sampling beam splitter in $E_{d d 1}$ and mixing $E_{d d 1}$ with $E_{b b 1}$, it then is possible to interchange the "bright" and "dark" outputs merely by adjusting the phase shifts applied to the inputs $E_{2}$ and $E_{4}$ (which interchanges the bright and dark outputs on the pairwise nullers.) The difference between the two resulting estimates of the leakage can provide a diagnostic that could be used for further calibration and/or system alignment.

\section{CONCLUSION}

We discuss the concept of coherent calibration of an interferometric null and its application to the Terrestrial Planet Finder instrument. We find that such an approach to calibration greatly relaxes the required levels of electric field matching, and associated stability requirements. This should improve instrument performance, particularly at shorter wavelengths where the effects of path length control limitations are most severe.

We are grateful to M. Colavita, O. Lay, and D. Kaplan for helpful comments during manuscript preparation. Part of the work described in this paper was performed at the Jet Propulsion Laboratory under contract with the National Aeronautics and Space Administration. B. F. L. acknowledges support from a Pappalardo Fellowship in Physics.
Bracewell, R. N. 1978, Nature, 274, 780

Coulter, D. R. 2004, Proc. SPIE, 5487, 1207

Danchi, W. C., Deming, D., Kuchner, M. J., \& Seager, S. 2003, ApJ, 597, L57

Des Marais, D. J., et al. 2002, Astrobiology, 2, 153

Draper, D. W., Elias, N. M., Noecker, M. C., Dumont, P. J., Lay, O. P., \& Ware, B. 2006, AJ, 131, 1822

Guyon, O. 2004, ApJ, 615, 562

Kaltenegger, L., Karlsson, A., Fridlund, M., \& Absil, O. 2003, in Earths: $D A R W I N / T P F$ and the Search for Extrasolar Terrestrial Planets (ESA SP-539; Noordwijk: ESA), 459
REFERENCES

Lay, O. P. 2004, Appl. Opt., 43, 6100 2005, Appl. Opt., 44, 5859

Noecker, M. C. 1999, in ASP Conf. Ser. 164, Ultraviolet-Optical Space Astronomy Beyond HST, ed. J. A. Morse, J. M. Shull, \& A. L. Kinney (San Francisco: ASP), 249

Wallace, J. K., et al. 2004, Proc. SPIE, 5491, 862

Woolf, N., \& Angel, J. R. 1998, ARA\&A, 36, 507 